\title{
Tunable and adjustable broadband RF photonic fractional Hilbert transformer based on a Kerr soliton crystal optical microcomb
}

\section{Mengxi Tan}

Swinburne University of Technology

\section{Xingyuan Xu}

Monash University

David Moss ( $\nabla$ dmoss@swin.edu.au )

Swinburne University of Technology https://orcid.org/0000-0001-5195-1744

\section{Research Article}

Keywords: Kerr frequency comb, Hilbert transform, integrated optics, all-optical signal processing

Posted Date: April 19th, 2021

DOI: https://doi.org/10.21203/rs.3.rs-406846/v1

License: (c) (i) This work is licensed under a Creative Commons Attribution 4.0 International License.

Read Full License 


\title{
Tunable and adjustable broadband RF photonic fractional Hilbert transformer based on a Kerr soliton crystal optical microcomb
}

\author{
M. Tan, X. Xu, and David J. Moss
}

\begin{abstract}
We demonstrate an RF photonic fractional Hilbert transformer based on an integrated Kerr microcomb source featuring a record low free spectral range of 49 GHz. By programming and shaping the comb lines according to calculated tap weights for up to 39 wavelengths across the $\mathrm{C}$-band, we achieve tunable bandwidths ranging from 1.2 to $15.3 \mathrm{GHz}$ as well as variable center frequencies from baseband to $9.5 \mathrm{GHz}$, for both standard integral and arbitrary fractional orders. We experimentally characterize the RF amplitude and phase response of the tunable bandpass and lowpass Hilbert transformers with 90 and 45-degree phase shifts. The experimental results show good agreement with theory, confirming the effectiveness of our approach as a powerful way to implement standard and fractional order Hilbert transformers with broad and variable bandwidths and center frequencies, with high reconfigurability and greatly reduced size and complexity.
\end{abstract}

Keywords-Kerr frequency comb, Hilbert transform, integrated optics, all-optical signal processing.

\section{INTRODUCTION}

$\mathrm{T}$ he Hilbert transform is a fundamental mathematical function for signal processing systems. It has found wide applications in radar systems, signal sideband modulators, measurement systems, speech processing, signal sampling, and many others [1-3]. Fractional Hilbert transforms can meet specific requirements for secure single sideband communications [2] as well as hardware keys [3] since they provide an additional degree of freedom in terms of a variable phase shift. In practical applications such as multiplexing and demultiplexing signals, analyzing individual sub-channel spectral components, etc., Hilbert transformers are typically realized as a truncated or windowed version of the ideal Hilbert transform impulse response [4-6]. Thus, Hilbert transformers that cover a wide range of different bandpass regions are in high demand. While electronic approaches to Hilbert transformers are subject to the well-known electronic bandwidth bottleneck [7], photonic RF techniques have attracted wide interest due to their numerous advantages, including optical bandwidths that can reach 10 's of $\mathrm{THz}$, a strong immunity to electromagnetic interference, and low transmission loss.

A significant amount of research has been devoted to

M. Tan, and D. J. Moss are with the Optical Sciences Centre, Swinburne University of Technology, Hawthorn, VIC 3122, Australia. (Corresponding email: $\underline{\text { dmoss@swin.edu.au). }}$ developing and extending the capability of photonic RF Hilbert transformers, including the use of free-space optics [3], although many approaches suffer from a tradeoff between performance and system complexity. Photonic approaches for both standard and fractional Hilbert transforms offer broad operational bandwidths through the use of novel approaches such as fibre Bragg gratings [8-14], macroing / microdisk resonators $[15,16]$, and integrated reconfigurable microwave processors [17]. However, most of these approaches focus only on generating the Hilbert transform of the complex optical field, and not the actual RF signal. To implement highly reconfigurable RF photonic Hilbert transformers, transversal schemes have been investigated. Fractional Hilbert transformers based on transversal structures have been demonstrated [18, 19] that offer high reconfigurability. However, methods that employ multiple discrete laser sources present limitations in the overall system footprint, processing performance, and the potential for full monolithic integration.

Recently, [20-39] microcombs have attracted significant interest as a fundamentally powerful tool for microwave processing, due to their ability to offer a large number of coherent wavelengths from a single device, thereby increasing the capacity of communications systems [40-42], for example. Further, they have enabled the processing of RF spectra for a wide range of advanced signal processing functions [43-68] as well as neural networks [69-71]. Previously, [43] we reported a Hilbert transformer based on a $200 \mathrm{GHz}$ spaced microcomb generating 20 wavelengths in the $\mathrm{C}$-band that achieved record performance in terms of its RF bandwidth (5 octaves). Subsequently, [63] we demonstrated a fractional Hilbert transformer with a performance in RF bandwidth ranging from 5 to 9 octaves, depending on the fractional order, by using 17 taps, or wavelengths, from a soliton crystal microcomb.

Here, we report a highly reconfigurable and versatile fractional Hilbert transformer based on a soliton crystal Kerr microcomb with a $49 \mathrm{GHz}$ comb spacing. The device has the ability to switch both its bandwidth and center frequency simultaneously and independently. This versatility, together with its high performance, is enabled by the use of $39 \mathrm{comb}$ lines generated by the microcomb over the C-band, representing more than twice the number of wavelengths, or taps, used in our previous work [63]. We experimentally demonstrate a Hilbert transformer with an RF amplitude and phase response at

$\mathrm{X} . \mathrm{Xu}$ is with the Electro-Photonics Laboratory, Department of Electrical and Computer System Engineering, Monash University, Clayton, 3800 VIC, Australia 


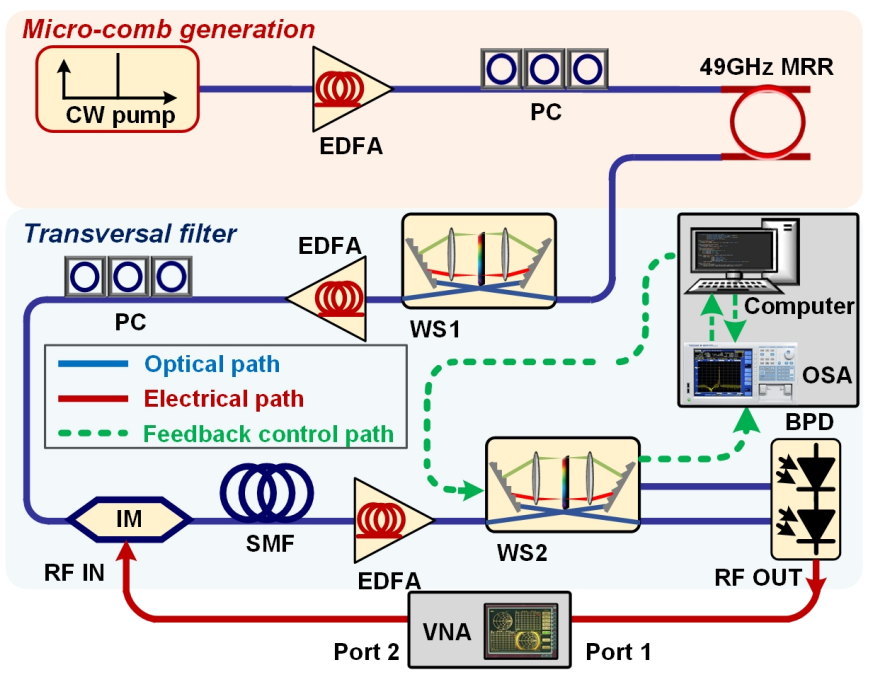

Fig. 1. Schematic diagram of fractional Hilbert transformer based on an integrated soliton crystal Kerr microcomb source. EDFA: erbium-doped fiber amplifier. PC: polarization controller. MRR: micro-ring resonator. WS: WaveShaper. IM: Intensity modulator. SMF: single mode fiber. OSA: optical spectrum analyzer. BPD: Balanced photodetector. VNA: vector network

bandwidths ranging from 1.2 to $15.3 \mathrm{GHz}$, together with center frequencies varying from baseband to $9.5 \mathrm{GHz}$. The experimental results agree well with the theory, confirming the feasibility of our approach towards the realization of high-speed reconfigurable Hilbert transformers with reduced footprint, lower complexity, and potentially reduced cost.

\section{PRINCIPAL OF OPERATION}

The spectral transfer function of a general fractional Hilbert transformer is given by $[62,71]$ :

$$
H_{P}(\omega)=\left\{\begin{array}{c}
e^{-j \varphi}, \text { if } 0 \leq \omega<\pi \\
e^{j \varphi}, \text { if }-\pi \leq \omega<0
\end{array}\right.
$$

where $j=\sqrt{ }-1, \varphi=P \times \pi / 2$ denotes the phase shift, $P$ is the fractional order (when $P=1$, it becomes a standard Hilbert transformer). The corresponding impulse response is given by a continuous hyperbolic function:

$$
h_{P}(t)=\left\{\begin{array}{c}
\frac{1}{\pi t}, t \neq 0 \\
\cot (\varphi), t=0
\end{array}\right.
$$

This hyperbolic function is truncated and sampled in time with discrete taps for digital implementation. The null frequency is given by:

$$
f_{c}=1 / \Delta t
$$

where $\Delta t$ denotes the sample spacing. The coefficient of the tap at $t=0$ can be adjusted to achieve a tunable fractional order [3]. The normalized power of each comb line is:

$$
P_{n}=\frac{1}{\pi\left|n-\frac{N}{2}+0.5\right|}
$$

where $N$ is the number of comb lines, or taps, and $n=0,1,2, \ldots$, $N-1$ is the comb index.
In order to scale the bandwidth of the standard and fractional Hilbert transformer, we multiplied the corresponding impulse response of the spectral transfer function of the Hilbert transformer with a cosine function to vary the operation bandwidth (designed by the Remez algorithm [72]). Hence, the resulting discrete impulse response becomes:

$$
h_{T B W H T}(n)=P_{n} \cdot \cos \left(2 \pi n \cdot f_{B W}\right)
$$

where $f_{\mathrm{BW}}$ is the scalable bandwidth. To further switch the center frequency of the Hilbert transformer, the tap coefficients were multiplied by a sine function to shift the RF transmission spectrum. The corresponding discrete impulse response is given by

$$
h_{T C F H T}(n)=P_{n} \cdot \sin \left(2 \pi n \cdot f_{B W}\right)
$$

Here, we use this transversal approach to achieve Hilbert transformers with both variable bandwidths as well as RF centre frequencies. The transfer function of the transversal structure [74-84] can be described as:

$$
F(\omega)=\sum_{n-0}^{M-1} h(n) e^{-j \omega n T}
$$

where $M$ is the number of taps, $\omega$ is the RF angular frequency, $T$ is the time delay between adjacent taps, and $h(n)$ is the tap coefficient of the $n^{\text {th }}$ tap.

Figure 1 illustrates the schematic diagram of the microcombbased RF photonic Hilbert transformer. The microcomb was generated by pumping a nonlinear high-Q MRR, fabricated on a high-index doped silica glass platform, with a $\mathrm{CW}$ laser, amplified with an erbium-doped fibre amplifier (EDFA), with the polarization state aligned to the TE mode of the MRR. When the pump wavelength was swept manually across one of the MRR's resonances and the pump power was high enough to generate sufficient parametric gain, optical parametric oscillation occurred, ultimately generating Kerr microcomb with a spacing equal to the free spectral range of the MRR. The generated microcomb was then spectrally flattened and 


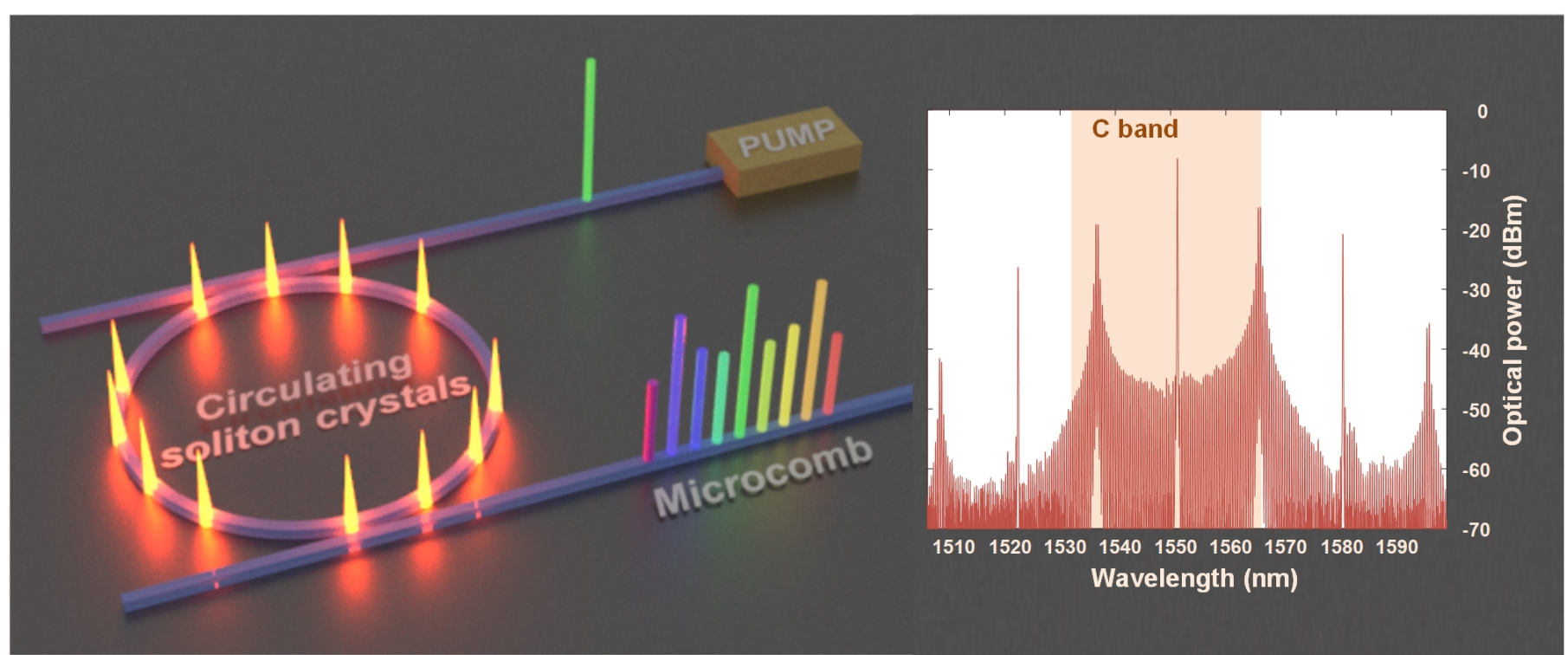

Fig. 2. Schematic illustration of the integrated MRR for generating the Kerr frequency comb and the optical spectrum of the generated soliton crystal combs with a 100-nm span.

modulated by the WaveShapers to achieve the designed tap weights.

Following this, the shaped comb lines were then imprinted with the RF signal via an intensity modulator, yielding replicas of the RF input waveform in the optical domain, transmitting the wavelength through a dispersive medium to acquire wavelength-dependent delays. The weighted and delayed signals were summed together upon photodetection and converted back into the RF domain.

\section{EXPERIMENTAL RESULTS}

The MRRs used here were fabricated on a platform based on Hydex glass [30, 33, 34, 38, 39] with CMOS compatible fabrication processes. First, Hydex films $(n=\sim 1.7$ at $1550 \mathrm{~nm})$ were deposited using plasma enhanced chemical vapour deposition (PECVD), then patterned by deep ultraviolet (UV) photolithography and etched via reactive ion etching [29] to achieve waveguides with very low surface roughness. Finally, an upper cladding layer composed of silica $(n=\sim 1.44$ at 1550 $\mathrm{nm}$ ) was deposited. We often employ a vertical coupling scheme between the ring resonator and bus, where the gap (approximately $200 \mathrm{~nm}$ ) can be controlled by film growth, which is much more accurate than using lithographic techniques. The advantages of our platform, particularly in relation to optical microcombs, include ultra-low linear loss $\left(\sim 0.06 \mathrm{~dB} \cdot \mathrm{cm}^{-1}\right)$, a moderately high optical nonlinear parameter $\left(\sim 233 \mathrm{~W}^{-1} \cdot \mathrm{km}^{-1}\right)$, and in particular, a negligible nonlinear loss - even up to extremely high intensities of about $25 \mathrm{GW} \cdot \mathrm{cm}^{2}$. As a result of the ultra-low loss of the platform, our MRR features narrow resonance linewidths corresponding to $\mathrm{Q}$ factors of up to $\sim 1.5$ million for the $49 \mathrm{GHz}$ MRR. The insertion loss of the through-port was as low as 0.5 to $1 \mathrm{~dB} /$ facet - a result of very efficient on-chip mode converters that allowed packaging the device with fibre pigtails. The radius of the MRR was $\sim 592 \mu \mathrm{m}$, corresponding to an optical FSR of $0.393 \mathrm{~nm}$ or $48.9 \mathrm{GHz}$. This small FSR greatly increased the number of

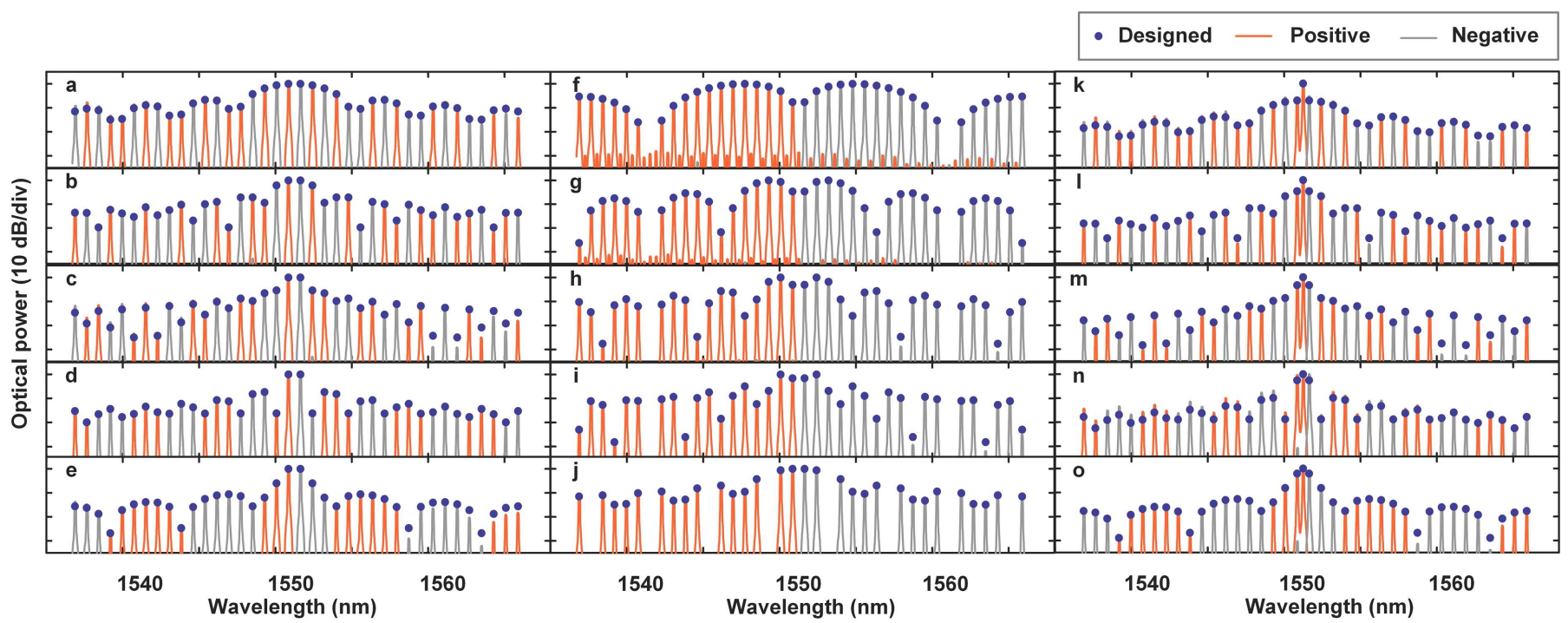

Fig. 3. Designed and measured optical spectra for (a) (e) Tunable bandpass Hilbert transformer with 90-degree phase shift. (f) (j) Tunable lowpass Hilbert transformer with 90-degree phase shift. (k) (o) Tunable bandpass fractional Hilbert transformer with 45-degree phase shift. 


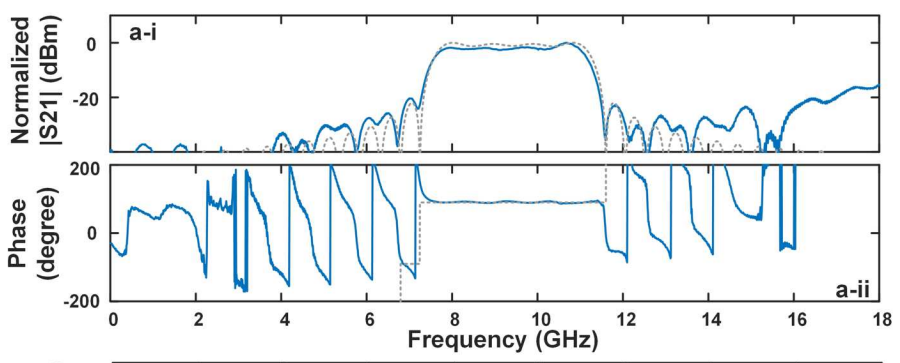

Bandpass Hilbert transformer 90 degree phase shift
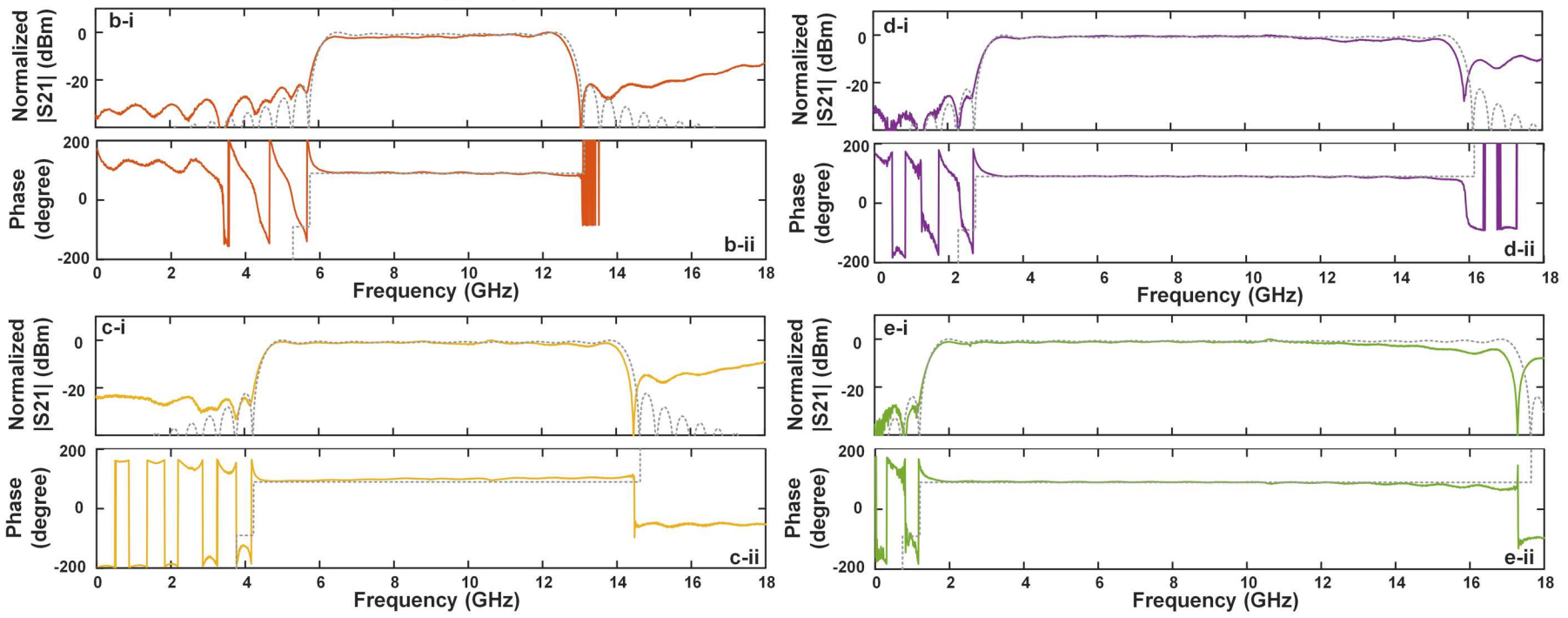

Fig. 4. Simulated (dashed curves) and experimental (solid curves) results of tunable bandpass Hilbert transformer with 90-degree phase shift.

wavelengths (channels) to as many as 75 over the $\mathrm{C}$ band, of which we used up to 39 wavelengths in this work, more than twice the number of wavelengths over our previous work [63].

To generate microcombs with the $49 \mathrm{GHz}$ device, the $\mathrm{CW}$ pump power was amplified to $\sim 30.5 \mathrm{dBm}$, and the wavelength swept from blue to red near one of the TE resonances of the MRR at $\sim 1553.2 \mathrm{~nm}$ [51]. When the detuning between the pump wavelength and cold resonance of the MRR became small enough, such that the intra-cavity power reached a threshold, modulation instability (MI) driven oscillation occurred [22]. Primary combs were then generated with the spacing determined by the MI gain peak - mainly a function of the dispersion and intra-cavity power. As the detuning was changed further, distinctive 'fingerprint' optical spectra eventually appeared (Fig. 2). The spectra are similar to what has been reported from spectral interference between tightly packed solitons in the cavity - so-called "soliton crystals" [26-28]. The soliton crystal states provided the lowest RF noise states of all our microcombs and have been used as the basis for an RF oscillator with low phase-noise [57].

The soliton crystal microcomb was first flattened via an optical spectral shaper (Finisar, WaveShaper 4000S) and then modulated with the RF input signal to multicast the RF waveform onto all wavelength channels simultaneously, yielding up to 75 replicas. These were then passed through $\sim 3.84 \mathrm{~km}$ of standard single mode fibre (SMF) to provide the progressive time delays between wavelengths. The dispersion of the SMF was $\sim 17.4 \mathrm{ps} / \mathrm{nm} / \mathrm{km}$, corresponding to a time delay $\Delta t=26.25$ ps between adjacent wavelengths. Next, the second WaveShaper accurately shaped the comb power according to the designed tap coefficients, with the shaped comb spectrum shown in Fig. 3. The wavelength channels for both positive (solid red line) and negative (solid gray line) taps were separately measured by an optical spectrum analyser (OSA), achieving good agreement with theory (blue dot). Finally, the weighted and delayed replicas were combined and converted back into the RF domain via a balanced photodetector (Finisar BPDV2150R).

The system RF frequency response was characterized with a calibrated vector network analyser (VNA, Agilent MS4644B) to measure the RF transmission and phase response. Fig. 4 (ae) presents the simulated (dashed curves) and measured (solid curves) RF frequency response for both the magnitude and phase of the standard Hilbert transformer with variable bandwidths ranging from 3.4 to $15.3 \mathrm{GHz}$. The centre frequency of the Hilbert transformer was set into half of the FSR RF, which was $19 / 2=9.5 \mathrm{GHz}$ in our case.

Figure 5 shows the measured results for the RF amplitude and phase response of the lowpass Hilbert transformer, showing tunable bandwidths ranging from 1.2 to $7.1 \mathrm{GHz}$ that match closely with the simulated results. We also performed a demonstration of a fractional Hilbert transformer with switchable RF bandwidths ranging from 3.5 to $15.2 \mathrm{GHz}$. The simulated and measured RF amplitude and phase responses are shown in Fig. 6. We achieved a fractional order of 0.5, which corresponds to a 45-degree phase shift. We used up to 39 wavelengths or taps, which represents an increase of a factor of two from our previous work. This resulted in a significant reduction in the overall root-mean-square error (RMSE) as well as an increase in the potential operational bandwidth, as was 

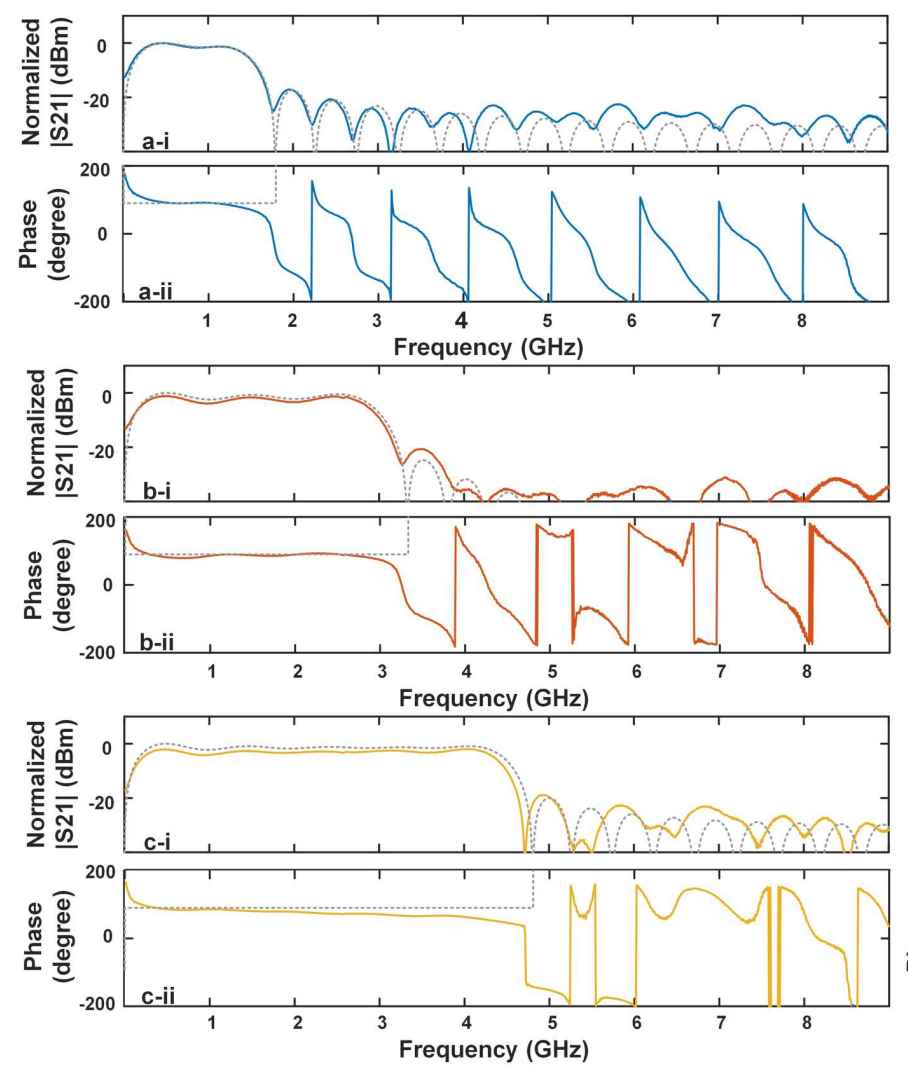

Fig. 5. Simulated (dashed curves) and experimental (solid curves) results of tunable lowpass Hilbert transformer with 90-degree phase shift.
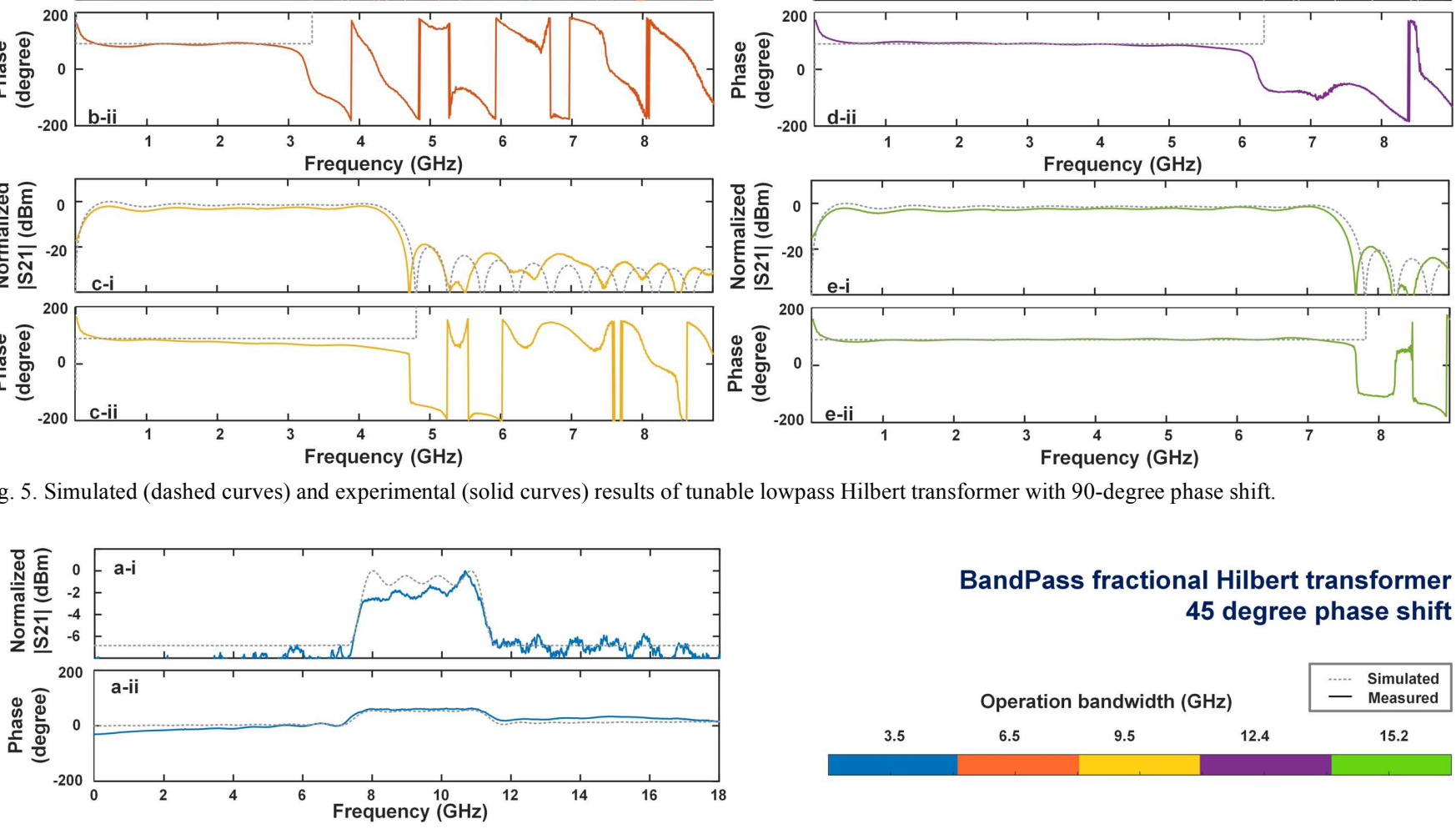

90 degree phase shift

BandPass fractional Hilbert transformer 45 degree phase shift

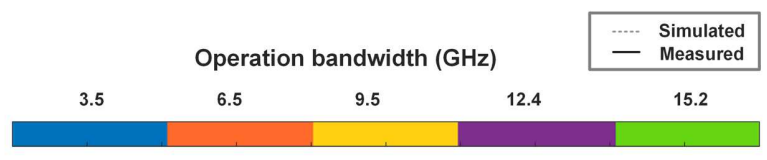

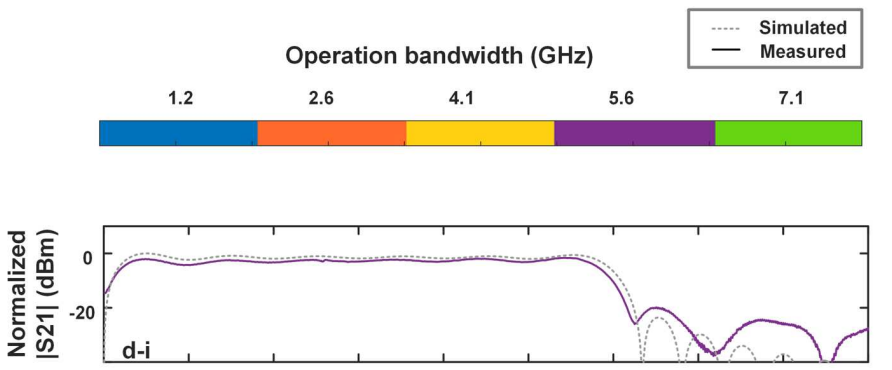
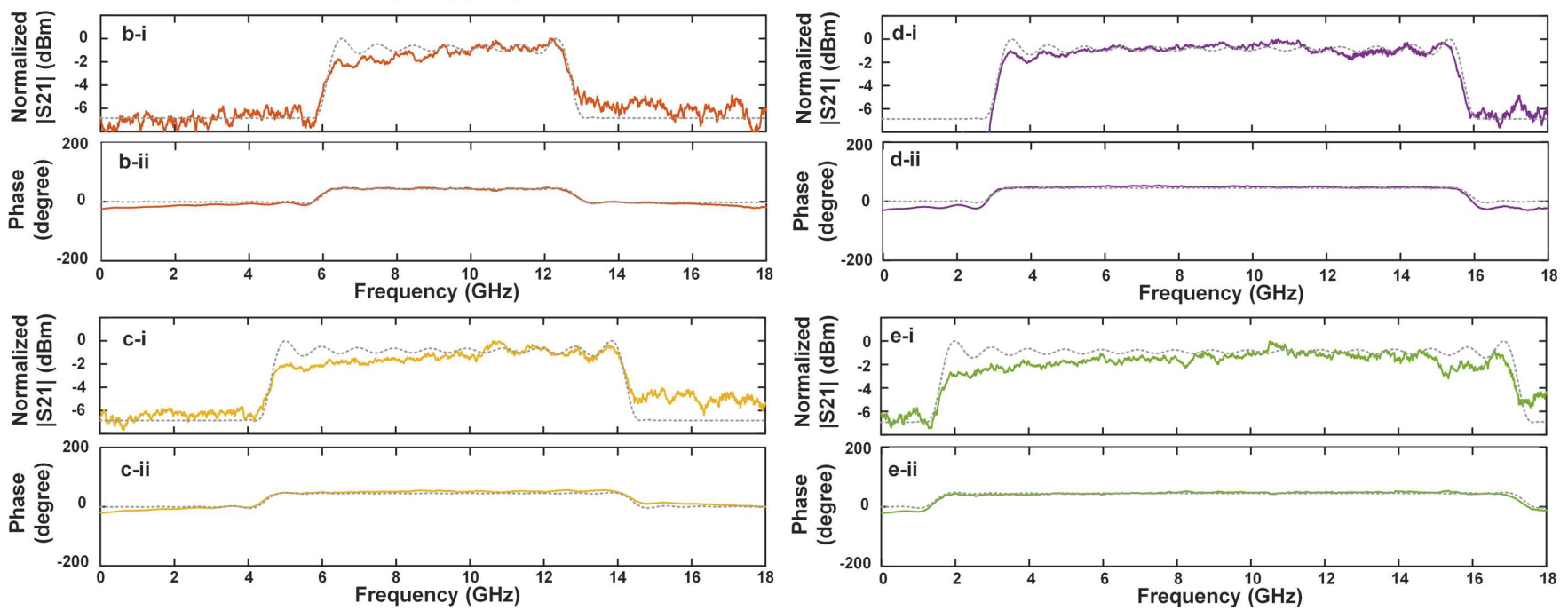

Fig. 6. Simulated (dashed curves) and experimental (solid curves) results of tunable bandpass fractional Hilbert transformer with 45-degree phase shift.

predicted [63]. Note, in order to achieve Hilbert transforms with tunable fractional orders we use an additional tap in the middle of the comb lines that we adjust in weight, resulting in an effective tap spacing of twice the FSR: $49 \times 2=98 \mathrm{GHz}$. Hence, for the fractional Hilbert transformer this resulted in 39 of the 75 comb lines generated across the $\mathrm{C}$-band being available. For consistency we used the same number of taps for the standard Hilbert transformer as well. 


\section{CONCLUSION}

We demonstrate a broadband RF photonic Hilbert transformer with variable bandwidths as well as RF center frequency, based on a Kerr soliton crystal microcomb. Up to 39 wavelengths, or taps were used of the 75 that were generated, resulting in a tunable bandwidth ranging from 1.2 to $15.3 \mathrm{GHz}$ as well as a switchable center frequency from baseband to 9.5 $\mathrm{GHz}$, achieved by programming and adjusting the tap weights. Microcombs are a powerful approach to achieving a fully integrated photonic signal processor for future ultra-high-speed RF systems.

\section{REFERENCES}

[1] S. L. Hahn, Transforms and Applications Handbook, A. D. Poularikas, Ed., 3rd ed. Boca Raton, FL: CRC Press, 2010, ch. 7.

[2] L. Moura, "Radio Frequency Implementation of the Fractional Hilbert Transform with Transversal Filters," Circuits, Systems \& Signal Processing., vol. 26, no. 3, pp. 407-417, Jun. 2007.

[3] A. W. Lohmann, D. Mendlovic, and Z. Zalevsky, "Fractional Hilbert transform," Opt. Lett., vol. 21, no. 4, pp. 281-283, Feb. 1996.

[4] V. K. Pedditi, and R. Kumaresan, "Bandpass phase shifter and analytic signal generator," Signal Processing, vol. 125, pp. 216-220, 2016.

[5] P. P. Bazargani, M. R. Fernandez-Ruiz, and J. Azana, "Tunable optical filter using photonic hilbert transformation," Optics InfoBase Conference Papers, 2013.

[6] H. P. Bazargani, M. R. Fernández-Ruiz, and J. Azaña, "Tunable, nondispersive optical filter using photonic Hilbert transformation," Optics Letters, vol. 30, no. 17, pp. 5232-5235, 2014.

[7] C. D. Holdenried, J. W. Haslett, and B. Davies, "A fully integrated 10$\mathrm{Gb} / \mathrm{s}$ tapped delay Hilbert transformer for optical single sideband," IEEE Microw. Wireless Compon. Lett., vol. 15, no. 5, pp. 303-305, May 2005.

[8] H. Emami, N. Sarkhosh, L. A. Bui, and A. Mitchell, "Wideband RF photonic in-phase and quadrature-phase generation," Opt. Lett, vol. 33, no. 2, pp. 98-100, Jan. 2008.

[9] M. Li, and J. P. Yao, "All-fiber temporal photonic fractional Hilbert transformer based on a directly designed fiber Bragg grating," Opt. Lett., vol. 35, no. 2, pp. 223-225, Jan. 2010

[10] M. Li, and J. P. Yao, "Experimental Demonstration of a Wideband Photonic Temporal Hilbert Transformer Based on a Single Fiber Bragg Grating," IEEE Photon. Technol. Lett., vol. 22, no. 21 pp. 1559-1561, Nov. 2010.

[11] M. H. Asghari, and J. Azana, "All-optical Hilbert transformer based on a single phase-shifted fiber Bragg grating: design and analysis," Opt. Lett., vol. 34, no. 3, pp. 334-336, Feb. 2009.

[12] T. Yang, J. Dong, L. Liu, S. Liao, S. Tan, L. Shi, D. Gao, and X. Zhang, "Experimental observation of optical differentiation and optical Hilbert transformation using a single SOI microdisk chip," Sci. Rep., vol. 4, pp. 3960,2014

[13] Z. Zhang et al., "Wideband and continuously-tunable fractional photonic Hilbert transformer based on a single high-birefringence planar Bragg grating," Opt. Express, vol. 26, pp. 20450-20458, 2018.

[14] C. Sima, J. C. Gates, C. Holmes, P. L. Mennea, M. N. Zervas, and P. G. R. Smith, "Terahertz bandwidth photonic Hilbert transformers based on synthesized planar Bragg grating fabrication," Opt. Lett., vol. 38, no. 17, pp. 3448-3451, Sep. 2013.

[15] L. Zhuang, M. R. Khan, W. Beeker, A. Leinse, R. Heideman, and C. Roeloffzen, "Novel microwave photonic fractional Hilbert transformer using a ring resonator-based optical all-pass filter," Opt. Exp., vol. 20, no. 24, pp. 26499-26510, Nov. 2012.

[16] H. Shahoei, P. Dumais, and J. P. Yao, "Continuously tunable photonic fractional Hilbert transformer using a high-contrast germanium-doped silica-on-silicon microring resonator," Opt. Lett., vol. 39, no. 9, pp. 27782781, May 2014.

[17] W. Liu et al., "A fully reconfigurable photonic integrated signal processor," Nature Photon., vol. 10, no. 3, pp. 190-196, 2016.

[18] Z. Li, Y. Han, H. Chi, X. Zhang, and J. P. Yao, "A continuously Tunable Microwave Fractional Hilbert Transformer Based on a Nonuniformly Spaced Photonic Microwave Delay-Line Filter," J. Lightwave Technol., vol. 30, no. 12, pp. 1948-1953, Jun. 2012.
[19] Z. Li, H. Chi, X. Zhang, and J. P. Yao, "A Continuously Tunable Microwave Fractional Hilbert Transformer Based on a Photonic Microwave Delay-Line Filter Using a Polarization Modulator," IEEE Photon. Technol. Lett., vol. 23, no. 22, pp. 1694-1696, Nov. 2011.

[20] M. Peccianti, M. Ferrera, L. Razzari, R. Morandotti, B. E. Little, S. T. $\mathrm{Chu}$, and D. J. Moss, "Subpicosecond optical pulse compression via an integrated nonlinear chirper," Opt. Exp., vol. 18, no. 8, pp. 7625-7633, Apr. 2010.

[21] D. Duchesne, M. Peccianti, M. R. E. Lamont, M. Ferrera, L. Razzari, F. Legare, R. Morandotti, S. Chu, B. E. Little, and D. J. Moss, "Supercontinuum generation in a high index doped silica glass spiral waveguide," Opt. Exp., vol. 18, no. 2, pp. 923-930, Jan. 2010.

[22] A. Pasquazi, M. Peccianti, L. Razzari, D. J. Moss, S. Coen, M. Erkintalo, Y. K. Chembo, T. Hansson, S. Wabnitz, P. Del'Haye, X. Xue, A. M. Weiner, and R. Morandotti, "Micro-combs: A novel generation of optical sources," Phys. Rep., vol. 729, pp. 1-81, Jan. 2018.

[23] P. Del'Haye, A. Schliesser, O. Arcizet, T. Wilken, R. Holzwarth, and T. J. Kippenberg, "Optical frequency comb generation from a monolithic microresonator," Nature, vol. 450, no. 20, pp. 1214-1217, Dec. 2007.

[24] T. J. Kippenberg, R. Holzwarth, and S. A. Diddams, "MicroresonatorBased Optical Frequency Combs," Science, vol. 332, pp. 555-559, Apr. 2011.

[25] J. S. Levy, A. Gondarenko, M. A. Foster, A. C. Turner-Foster, A. L. Gaeta, and M. Lipson, "CMOS-compatible multiple-wavelength oscillator for on-chip optical interconnects," Nature Photonics, vol. 4, no. 259, pp. 37-40, Dec. 2009.

[26] W. Wang, Z. Lu, W. Zhang, S. T. Chu, B. E. Little, L. Wang, X. Xie, M. Liu, Q. Yang, L. Wang, J. Zhao, G. Wang, Q. Sun, Y. Liu, Y. Wang, and W. Zhao, "Robust soliton crystals in a thermally controlled microresonator," Opt. Lett., vol. 43, no. 9, pp. 2004-2005, May. 2018.

[27] D. C. Cole, E. S. Lamb, P. Del'Haye, S. A. Diddams, and S. B. Papp, "Soliton crystals in Kerr resonators," Nature Photonics, vol. 11, pp. 671676, Oct. 2017

[28] M. Karpov, M. H. P. Pfeiffer, HGuo, W. Weng, J. Liu, T. J. Kippenberg, "Dynamics of soliton crystals in optical microresonators", Nature Physics vol. 15, no. 10, pp. 1071-1077, 2019.

[29] L. Razzari, D. Duchesne, M. Ferrera, R. Morandotti, S. Chu, B. E. Little and D. J. Moss, "CMOS-compatible integrated optical hyper-parametric oscillator," Nature Photonics, vol. 4, no. 1, pp. 41-45, Dec. 2009.

[30] D. J. Moss, R. Morandotti, A. L. Gaeta, and M. Lipson, "New CMOScompatible platforms based on silicon nitride and Hydex for nonlinear optics," Nature Photonics, vol. 7, no. 8, pp. 597-607, Jul. 2013.

[31] M. Ferrera, L. Razzari, D. Duchesne, R. Morandotti, Z. Yang, M. Liscidini, J. E. Sipe, S. Chu, B. E. Little, and D. J. Moss, "Low-power continuous-wave nonlinear optics in doped silica glass integrated waveguide structures," Nature Photonics, vol. 2, no. 12, pp. 737-740, Dec. 2008.

[32] H. Bao el al., "Laser cavity-soliton micro-Combs," Nature Photo., vol. 13, no. 6, pp. 384-389, 2019.

[33] A. Pasquazi, M. Peccianti, B. E. Little, S. T. Chu, D. J. Moss, and R. Morandotti, "Stable, dual mode, high repetition rate mode-locked laser based on a microring resonator," Opt. Exp., vol. 20, no. 24, pp. 2735527362, Nov. 2012

[34] A. Pasquazi, L. Caspani, M. Peccianti, M. Clerici, M. Ferrera, L. Razzari, D. Duchesne, B. E. Little, S. T. Chu, D. J. Moss, and R. Morandotti, "Self-locked optical parametric oscillation in a CMOS compatible microring resonator: a route to robust optical frequency comb generation on a chip," Opt. Exp., vol. 21, no. 11, pp. 13333-13341, Jun. 2013.

[35] A. Pasquazi, M. Peccianti, Y. Park, B. E. Little, S. T. Chu, R.Morandotti, J. Azaña, and D. J. Moss, "Sub-picosecond phase-sensitive optical pulse characterization on a chip", Nature Photonics, Vol. 5, No. 10, pp. 618 623 (2011).

[36] V. T.-Company, and A. M. Weiner, "Optical frequency comb technology for ultra-broadband radio-frequency photonics," Laser Photonics Rev., vol. 8, no. 3, pp. 368-393, 2014.

[37] M. Peccianti, A. Pasquazi, Y. Park, B. E. Little, S. T. Chu, D. J. Moss, and R. Morandotti, "Demonstration of a stable ultrafast laser based on a nonlinear microcavity," Nature Commun., vol. 3, 765, Apr. 3, 2012.

[38] L. D. Lauro, J. Li, D. J. Moss, R. Morandotti, S. T. Chu, M. Peccianti, and A. Pasquazi, "Parametric control of thermal self-pulsation in microcavities," Opt. Lett. vol. 42, no. 17, pp. 3407-3410, Aug. 2017.

[39] H. Bao, A. Cooper, S. T. Chu, D. J. Moss, R. Morandotti, B. E. Little, M. Peccianti, and A. Pasquazi, "Type-II micro-comb generation in a filter- 
driven four wave mixing laser [Invited]," Photonics Research, vol. 6, no. 5, pp. B67-B73, May 2018.

[40] P. Marin-Palomo, el al., "Microresonator-based solitons for massively parallel coherent optical communication", Nature, vol. 546, no. 7657, pp. 274, 2017.

[41] J. Pfeifle, V. Brasch, M. Lauermann, Y. Yu, D. Wegner, T. Herr, K. Hartinger, et al., "Coherent terabit communications with microresonator Kerr frequency combs", Nature Photonics, vol. 8, no. 5, pp. 375-380, 2014.

[42] B. Corcoran, et al., "Ultra-dense optical data transmission over standard fibre with a single chip source", Nature Communications, vol. 11, no. 1 , pp. 1-7, 2020.

[43] T. G. Nguyen, M. Shoeiby, S. T. Chu, B. E. Little, R. Morandotti, A. Mitchell, and D. J. Moss, "Integrated frequency comb source based Hilbert transformer for wideband microwave photonic phase analysis," Opt. Exp., vol. 23, no. 17, Aug. 2015.

[44] X. Xue, et al., "Programmable single-bandpass photonic RF filter based on a Kerr comb from a microring," Journal of Lightwave Technol., vol. 32, no. 20, pp. 3557-3565, 2014.

[45] X. Xu, J. Wu, M. Shoeiby, T. G. Nguyen, S. T. Chu, B. E. Little, R. Morandotti, A. Mitchell, and D. J. Moss, "Reconfigurable broadband microwave photonic intensity differentiator based on an integrated optical frequency comb source," APL Photonics, vol. 2, no. 9, pp. 096104 2017.

[46] X. Xu, M. Tan, J. Wu, R. Morandotti, A. Mitchell, and D. J. Moss, "Microcomb-based photonic RF signal processing", IEEE Photonics Technology Letters, vol. 31, no. 23, pp. 1854-1857, 2019.

[47] X. Xu, J. Wu, T. G. Nguyen, M. Shoeiby, S. T. Chu, B. E. Little, R. Morandotti, A. Mitchell, and D. J. Moss, "Advanced RF and microwave functions based on an integrated optical frequency comb source," Opt. Exp., vol. 26, no. 3, pp. 2569-2583, Feb. 2018.

[48] X. Xue, et al., "Microcomb-based true-time-delay network for microwave beamforming with arbitrary beam pattern control," Journal of Lightwave Technology, vol. 36, no. 12, pp. 2312-2321, 2018.

[49] J. Wu, X. Xu, T. G. Nguyen, S. T. Chu, B. E. Little, R. Morandotti, A. Mitchell, and D. J. Moss, "RF Photonics: An Optical Microcombs' Perspective," IEEE J. Sel. Top. Quantum Electron., vol. 24, no. 4, pp. 6101020, Jul. 2018.

[50] X. Xu, J. Wu, T. G. Nguyen, S. T. Chu, B. E. Little, R. Morandotti, A. Mitchell, and D. J. Moss, "Broadband RF Channelizer Based on an Integrated Optical Frequency Kerr Comb Source," J. Lightwave Technol., vol. 36 , no. 19 , pp. 4519-4526, Oct. 2018.

[51] X. Xu, J. Wu, M. Tan, T. G. Nguyen, S. T. Chu, B. E. Little, R. Morandotti, A. Mitchell, and D. J. Moss, "Orthogonally Polarized RF Optical Signal Sideband Generation and Dual-Channel Equalization Based on an Integrated Microring Resonator," J. Lightw. Technol., vol. 36, no. 20, pp. 4808-4818, Oct. 2018.

[52] X. Xu, J. Wu, L. Jia, M. Tan, T. G. Nguyen, S. T. Chu, B. E. Little, R. Morandotti, A. Mitchell, and D. J. Moss, "Continuously tunable orthogonally polarized RF optical signal sideband generator based on micro-ring resonators," IOP J. Optics., vol. 20, no. 11, pp. 115701, Oct. 2018.

[53] X. Xu, J. Wu, T. G. Nguyen, T. Moein, S. T. Chu, B. E. Little, R. Morandotti, A. Mitchell, and D. J. Moss, "Photonic microwave true time delays for phased array antennas using a $49 \mathrm{GHz}$ FSR integrated optical micro-comb source [Invited]," Photonics Research, vol. 6, no. 5, pp. B30-B36, May 2018.

[54] X. Xu, M. Tan, J. Wu, T. G. Nguyen, S. T. Chu, B. E. Little, R. Morandotti, A. Mitchell, and D. J. Moss, "Advanced Adaptive Photonic RF Filters with 80 Taps Based on an Integrated Optical Micro-Comb Source," J. Lightwave Technol., vol. 37, no. 4, pp. 1288-1295, Feb. 2019.

[55] W. Liang, et al., "High spectral purity Kerr frequency comb radio frequency photonic oscillator," Nature Communications, vol. 6, pp. 7957 2015.

[56] J. Liu, et al., "Photonic microwave generation in the X-andK-band using integrated soliton microcombs," Nature Photonics, vol. 14, pp. 1-6, 2020

[57] X. Xu, et al., "Broadband microwave frequency conversion based on an integrated optical micro-comb source", Journal of Lightwave Technology, vol. 38, no. 2, pp. 332-338, 2020.

[58] M. Tan, et al., "Photonic RF and microwave filters based on $49 \mathrm{GHz}$ and $200 \mathrm{GHz}$ Kerr microcombs", Optics comm., vol. 465, Article: 125563 , 2020.

[59] X. Xu, et al., "Broadband photonic RF channelizer with 92 channels based on a soliton crystal microcomb," Journal of Lightwave Technology, vol. 38 , no. 18 , pp. 5116-5121, 2020.
[60] X. Xu, et al., "Photonic RF and Microwave Integrator Based on a Transversal Filter With Soliton Crystal Microcombs," IEEE Transactions on Circuits and Systems II: Express Briefs, vol. 67, no. 12, pp. 3582-3586, 2020.

[61] X. Xu, et al., "Photonic RF phase-encoded signal generation with a microcomb source", Journal of Lightwave Technology, vol. 38, no. 7, pp. $1722-1727,2020$.

[62] X. Xu, M. Tan, J. Wu, T. G. Nguyen, S. T. Chu, B. E. Little, R. Morandotti, A. Mitchell, and D. J. Moss, "High performance RF filters via bandwidth scaling with Kerr micro-combs," APL Photonics, vol. 4, no. 2 , pp. 026102, Feb. 2019.

[63] M. Tan, et al., "Microwave and RF photonic fractional Hilbert transformer based on a $50 \mathrm{GHz}$ Kerr micro-comb", Journal of Lightwave Technology, vol. 37, no. 24, pp. 6097-6104, 2019.

[64] M. Tan, et al., "RF and microwave fractional differentiator based on photonics", IEEE Transactions on Circuits and Systems II: Express Briefs, vol. 67, no. 11, pp. 2767-2771, 2020.

[65] M. Tan, et al., "Photonic RF arbitrary waveform generator based on a soliton crystal micro-comb source", Journal of Lightwave Technology, vol. 38, no. 22, pp. 6221-6226, 2020.

[66] M. Tan, et al., "RF and microwave photonic temporal signal processing with Kerr micro-combs", Advances in Physics: X, vol. 6, no. 1, pp. 18838946, 2021.

[67] M. Tan, et al., "Orthogonally polarized RF optical single sideband generation with integrated ring resonators." Journal of Semiconductors, vol. 42, pp. 1-14, 2021. DOI: 10.1088/1674-4926/42/4/041305.

[68] M. Tan, et al., "Photonic Radio Frequency Channelizers based on Kerr Optical Micro-combs", Journal of Semiconductors, Vol. 42, No. 4, 041302 (2021). DOI:10.1088/1674-4926/42/4/041302.

[69] X. Xu, et al., "Photonic perceptron based on a Kerr microcomb for scalable high speed optical neural networks", Laser and Photonics Reviews, vol. 14, no. 10, pp. 2000070, 2020.

[70] X. Xu, et al., "11 TOPS photonic convolutional accelerator for optical neural networks," Nature, vol. 589, no. 7840, pp. 44-51, 2021.

[71] J. Feldmann, et al., "Parallel convolutional processing using an integrated photonic tensor core," Nature, vol 589, no. 7840, pp. 52-58, 2021

[72] J. Yao, "Microwave Photonics," J. Lightw. Technol., vol. 27, no. 3, pp. 314-335, Feb. 2009.

[73] J. H. Mcclellan, T. W. Parks, and L. R. Rabiner, "Couputer-program for designing optimum FIR linear phase digital filters," IEEE Trans. Audio Electroacoust., vol. AU-21, no. 6, pp. 506-526, 1973.

[74] Z. J. Zhu, H. Chi, T. Jin, S. L. Zheng, X. F. Jin, and X. M. Zhang, "Allpositive-coefficient microwave photonic filter with rectangular response," Opt. Lett., vol. 42, no. 15, pp. 3012-3015, Aug. 2017.

[75] R. A. Minasian, "Ultra-Wideband and Adaptive Photonic Signal Processing of Microwave Signals," IEEE Journal of Quantum Electronics, vol. 52, no. 1, pp: 1-13, Jan. 2016.

[76] W. Wang, W. Zhang, Z. Lu, S. T. Chu, B. E. Little, Q. Yang, L. Wang, and W. Zhao, "Self-locked orthogonal polarized dual comb in a microresonator," Photonics Research, vol. 6, No. 5, pp. 363-367, May 2018.

[77] J. Capmany, J. Mora, I. Gasulla, J. Sancho, J. Lloret, and S. Sales, "Microwave Photonic Signal Processing," J. Lightwave Technol., vol. 31, no. 4, pp: 571-586, Feb. 2013.

[78] R. A. Minasian, "Photonic Signal Processing of Microwave Signals," IEEE Trans. Micro. Theory Tech., vol. 45, no. 8, pp. 1463-1466, Aug. 1997.

[79] J. Capmany, and D. Novak, "Microwave photonics combines two worlds," Nature Photon., vol. 1, pp. 319-330, Jun. 2007.

[80] J. Capmany, B. Ortega, and D. Pastor, "A Tutorial on Microwave Photonic Filters," J. Lightw. Technol., vol. 24, no. 1, pp. 201-229, Jan. 2006.

[81] R. C. Williamson, R. D. Esman, "RF Photonics," J. Lightw. Technol., vol. 26, no. 9, pp. 1145-1153, May 2008.

[82] Y. Liu, J. Hotten, A. Choudhary, B. J. Eggleton, "All-optimized integrated RF photonic notch filter," Opt. Lett., vol. 42, no. 22, pp. 46314634, Nov. 2017.

[83] C.-C. Tseng, and S.-C. Pei, "Design and Application of Discrete-Time Fractional Hilbert Transformer," IEEE Trans. Circuits Syst. II, Analog Digital Signal Process., vol, 47, no. 12, pp. 1529-1533, Dec. 2000.

[84] H. Emami, N. Sarkhosh, L. A. Bui, and A. Mitchell, "Wideband RF photonic in-phase and quatrature-phase generation," Opt. Lett., vol. 33, no. 2, pp. 98-100, Jan. 2008 


\section{Figures}

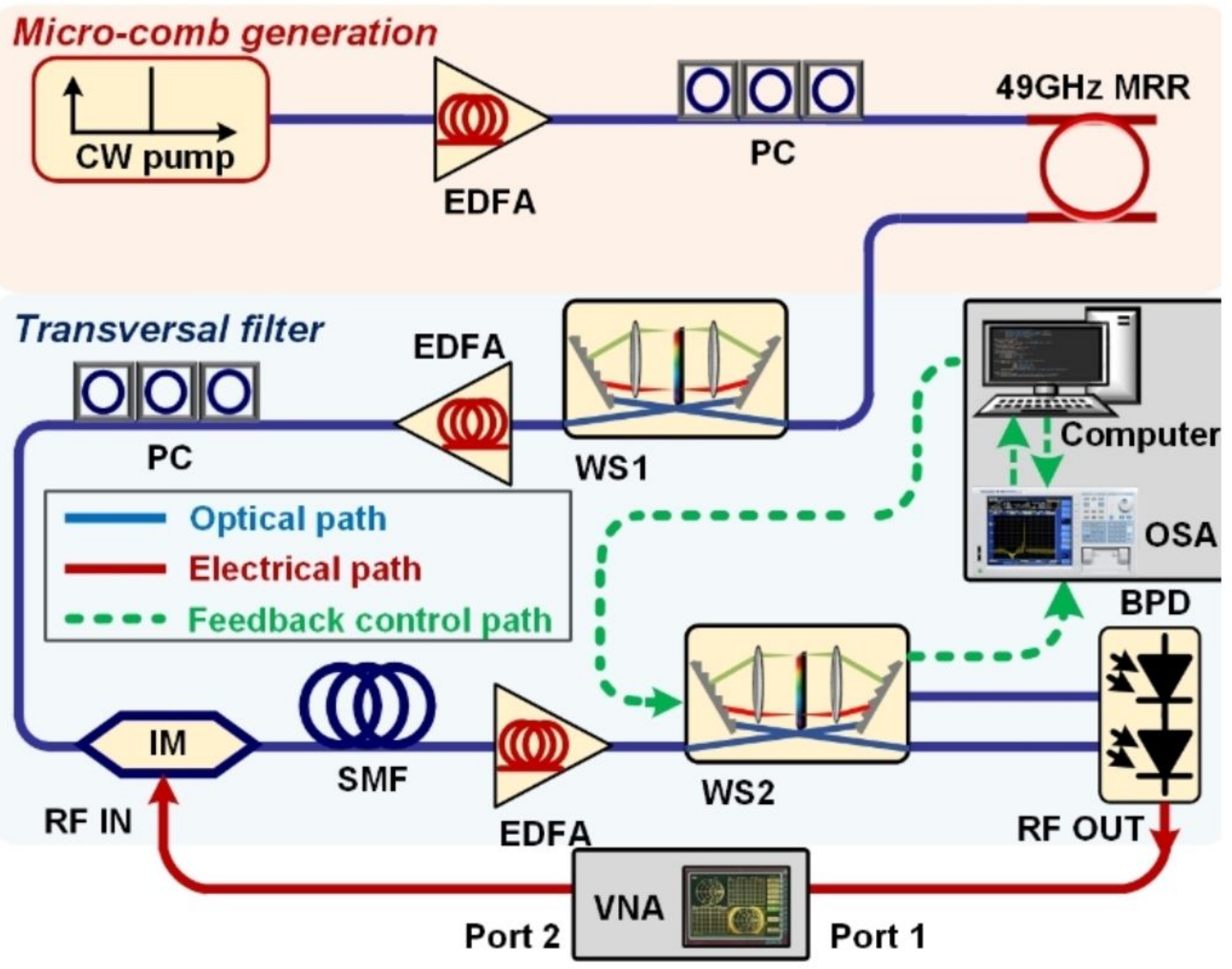

Fig. 1. Schematic diagram of fractional Hilbert transformer based on an integrated soliton crystal Kerr microcomb source. EDFA: erbium-doped fiber amplifier. PC: polarization controller. MRR: micro-ring resonator. WS: WaveShaper. IM: Intensity modulator. SMF: single mode fiber. OSA: optical spectrum analyzer. BPD: Balanced photodetector. VNA: vector network s...n......

Figure 1

Schematic diagram of fractional Hilbert transformer based on an integrated soliton crystal Kerr microcomb source. EDFA: erbium-doped fiber amplifier. PC: polarization controller. MRR: micro-ring resonator. WS: WaveShaper. IM: Intensity modulator. SMF: single mode fiber. OSA: optical spectrum analyzer. BPD: Balanced photodetector. VNA: vector network analyzer. 


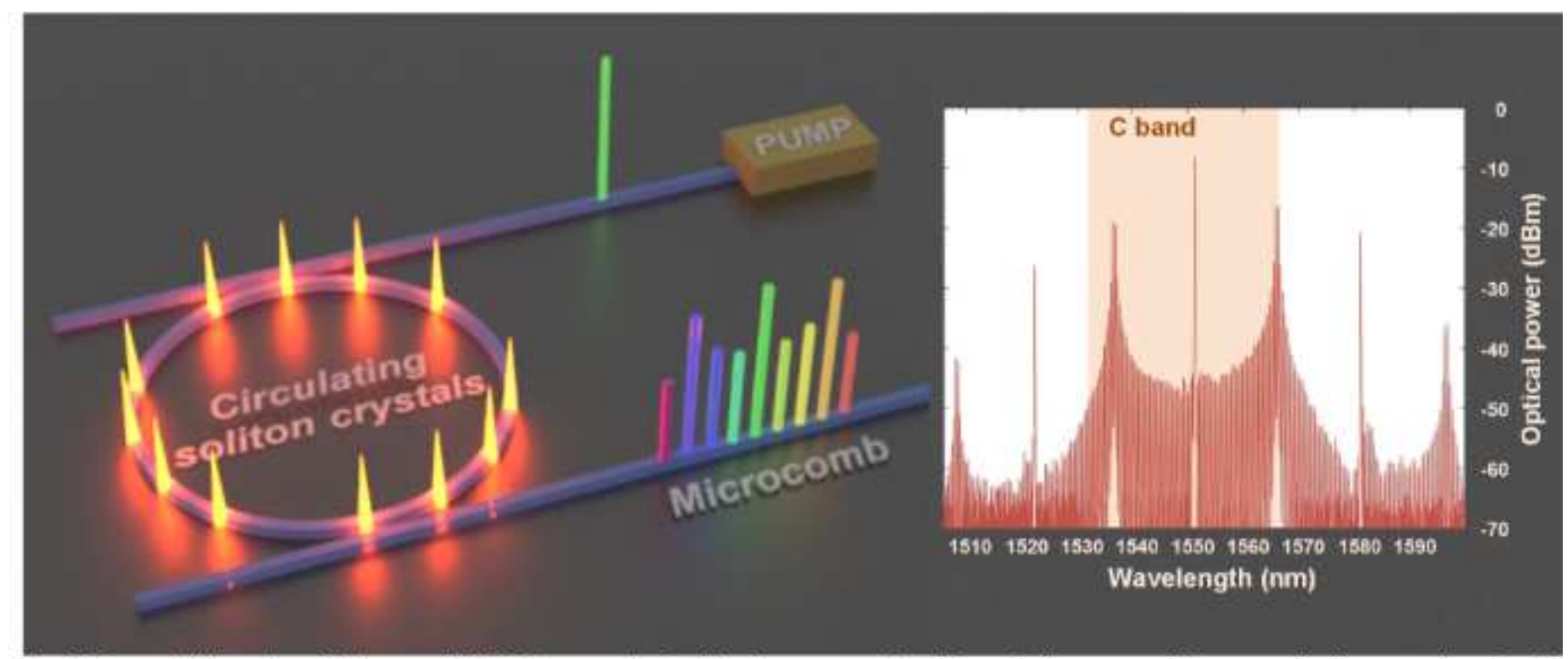

Fig. 2. Schematic illustration of the integratedMRR for generating the Ker frecquency comb and the optical spectrum of the generated soliton crystal combs with a $100-\mathrm{nm}$ span.

\section{Figure 2}

Schematic illustration of the integrated MRR for generating the Kerr frequency comb and the optical spectrum of the generated soliton crystal combs with a 100-nm span.

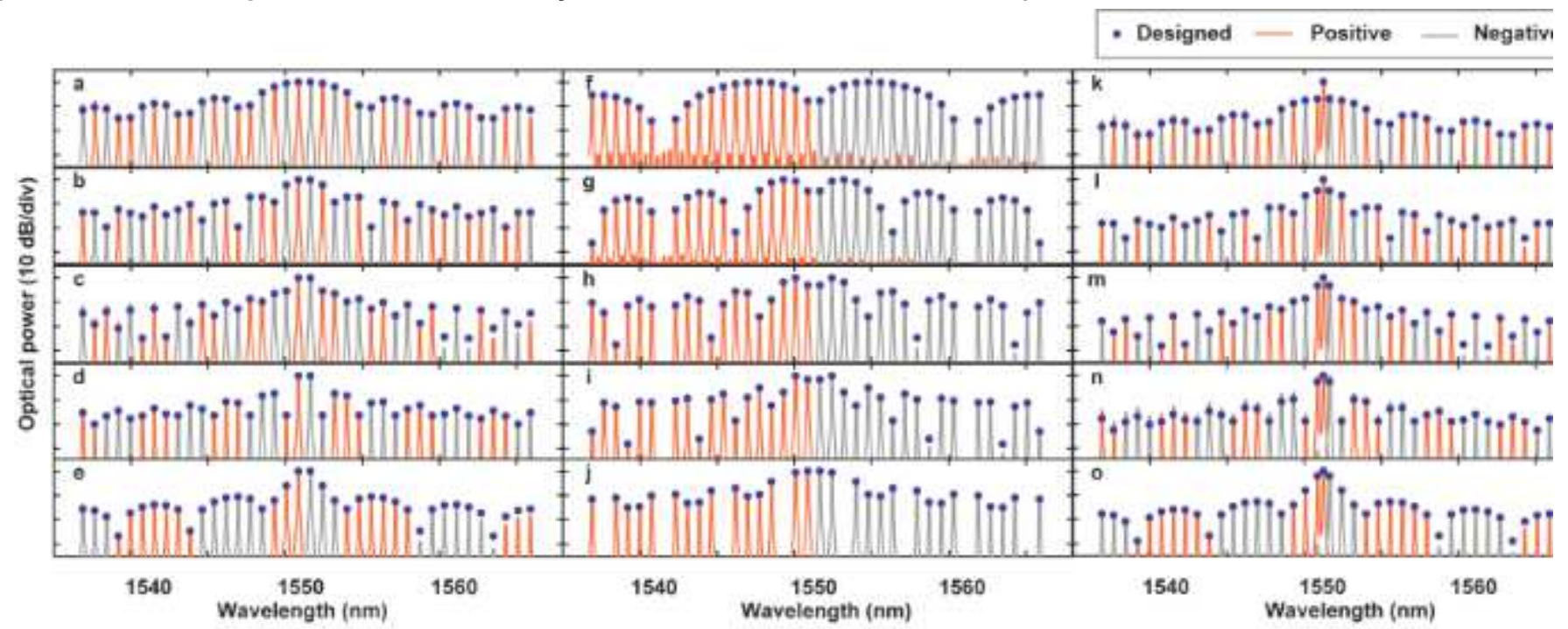

Fig. 3. Designed and messured optical spectra for (a) (e) Tunable bandpass Hilbert transformer with 90 -degree phase shift. (f) (j) Tumable lowpass Hilbert transformer with 90 -degree phase shift. (k) $\sim$ (o) Tunable bandpass fractional Hilbert transformer with 45 -degree phase shift.

\section{Figure 3}

Designed and measured optical spectra for (a) (e) Tunable bandpass Hilbert transformer with 90-degree phase shift. (f) (j) Tunable lowpass Hilbert transformer with 90-degree phase shift. (k) (o) Tunable bandpass fractional Hilbert transformer with 45-degree phase shift. 


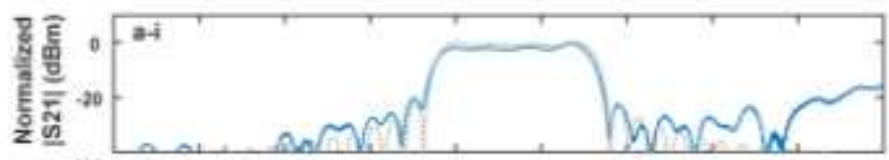

Bandpass Hilbert transformer 90 degree phase shift
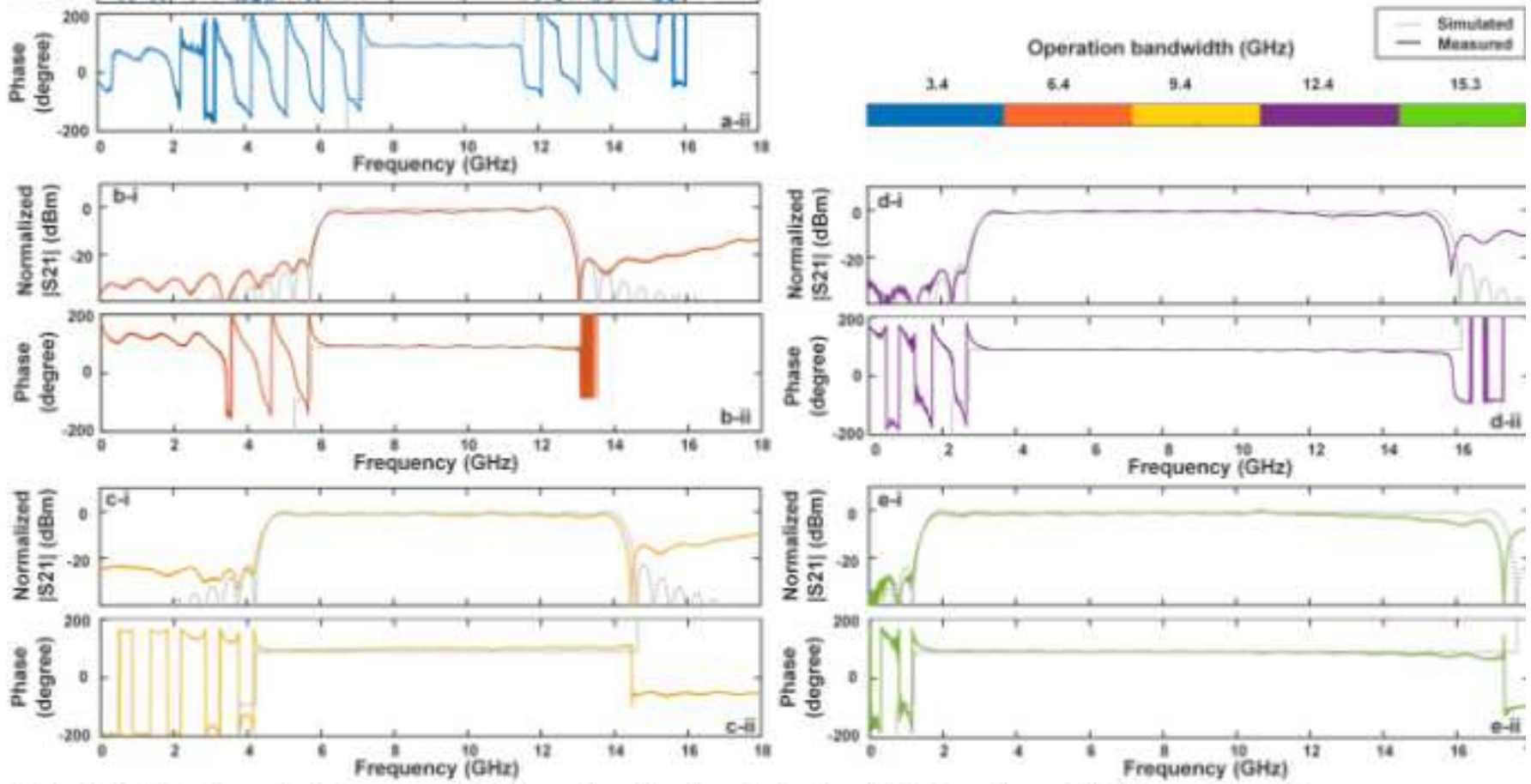

Fig. 4. Simulated (dashed curves) and experimental (solid curves) results of tunable bandpass Hilbert transformer with 90 -degree phase shift.

\section{Figure 4}

Simulated (dashed curves) and experimental (solid curves) results of tunable bandpass Hilbert transformer with 90-degree phase shift. 

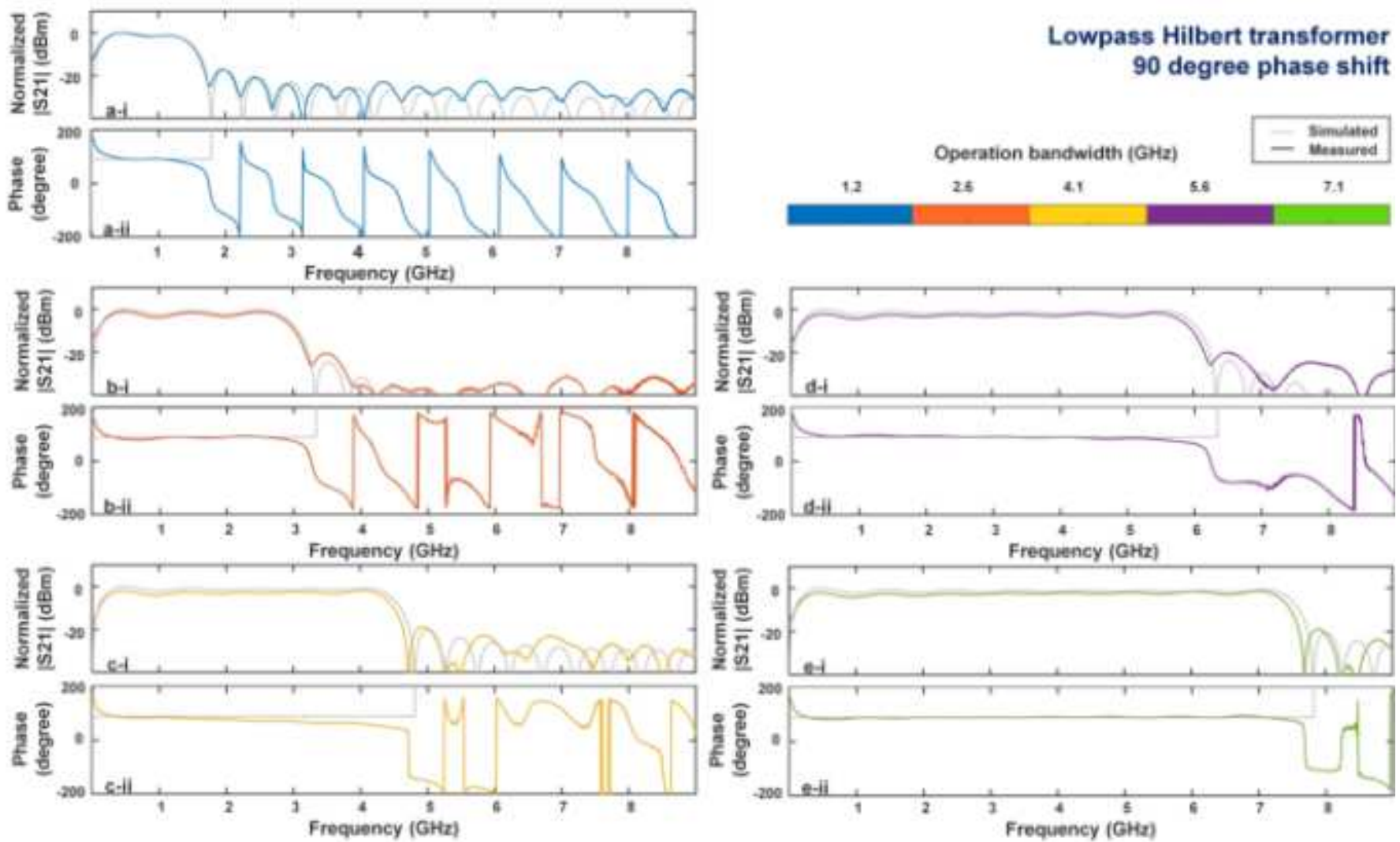

Fig. 5. Simulated (dashed curves) and experimental (solid curves) results of tumable lowpass Hilbert transformer with 90-degree phase shift.

\section{Figure 5}

Simulated (dashed curves) and experimental (solid curves) results of tunable lowpass Hilbert transformer with 90-degree phase shift. 


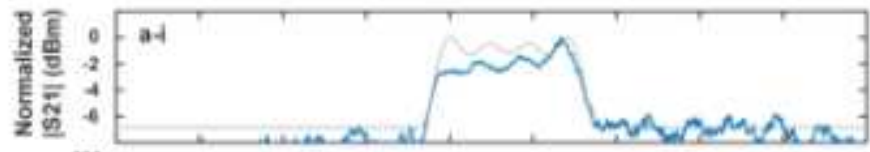

\section{BandPass fractional Hilbert transformer 45 degree phase shift}
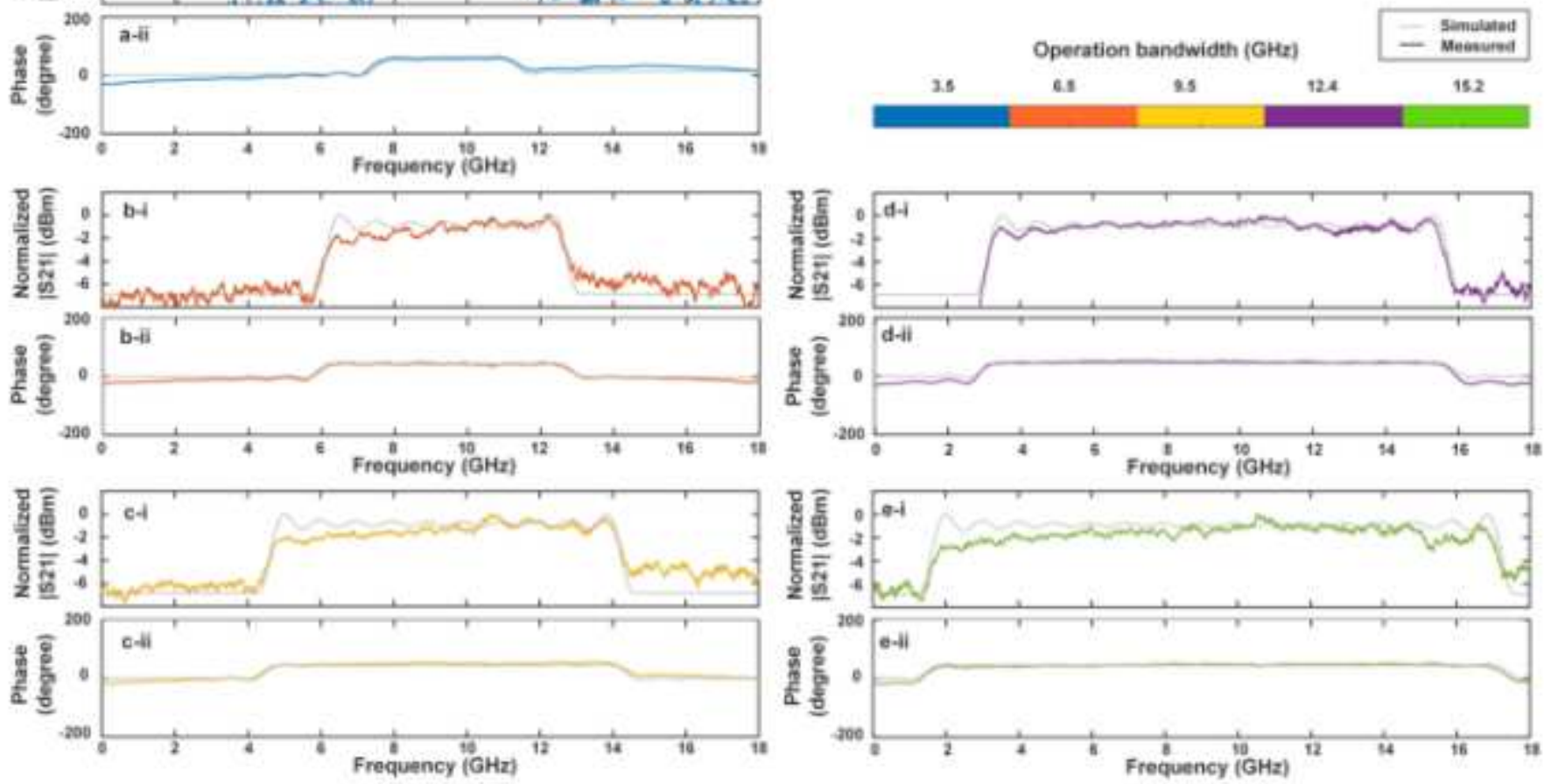

Fig. 6. Simulated (dashed curves) and experimental (solid curves) results of tuable bandpass fractional Hilbert transfomer with 45-degree phase shift.

\section{Figure 6}

Simulated (dashed curves) and experimental (solid curves) results of tunable bandpass fractional Hilbert transformer with 45-degree phase shift. 